\title{
Laterale thorakale Meningozele bei Skoliose und Neurofibromatose Typ I
}

Laterale thorakale Meningozelen sind in den meisten Fällen mit einer Neurofibromatose Typ I (NF 1) verbunden und gehen häufig mit einer Skoliose der Brustwirbelsäule (BWS) einher. Die NF 1 betrifft eine Vielzahl mesenchymaler Gewebe: Im Bereich der Wirbelsäule können Knochen, Dura, Nervenwurzeln und Rückenmark betroffen sein. Die Skoliose entsteht in der Regel als Folge einer Dysplasie der betroffenen Brustwirbel. Es bleibt aber unklar, ob eine Meningozele durch eine primäre Dysplasie der Meningen entlang des Foramen intervertebrale oder eine primäre Dysplasie der angrenzenden Wirbelbögen entsteht. Für unabhängige, koinzidente Veränderungen spricht, dass man bei Patienten mit NF 1 häufig anatomisch voneinander entfernte Dysplasien des Knochens und der Dura findet.

\section{Fallbericht}

Bei einer 32-jährigen Patientin mit bekannter Neurofibromatose Typ I wurde vor 18 Jahren eine rechts-konvexe Sko- liose der BWS mittels Harrington Instrumentarium versorgt. Über eine Meningozele wurde damals weder radiologisch noch intraoperativ berichtet. Seit einem Jahr klagte die Patientin über zunehmende Rückenschmerzen, teilweise so heftig, dass sie nicht mehr gehen konnte. Die neurologische Untersuchung war unauffällig. Röntgenaufnahmen der BWS wiesen eine flache, rechtskonvexe Skoliose auf mit einem Harrington-Stab zwischen BWK 4 und 10. Die Brustwirbel 5-8 waren dysplastisch (Abb.1). Auf der Übersichtsaufnahme des Thorax sah man eine halbkugelförmige, paravertebrale Raumforderung rechts intrathorakal (Abb. 2). Das Computertomogramm des Thorax zeigte eine zystische Erweiterung des Duralsackes rechts paravertebral durch laterale Defekte der betroffenen Wirbelkörper (Abb.3). Das Magnetresonanztomogramm (MRT) der BWS ließ wegen Metallartefakten keine eindeutige Abgrenzung von Rückenmark, Nervenwurzeln und Meningozele zu. Deswegen wurde eine Myelographie durchgeführt, wobei sich das Kontrastmittel in einer thorakalen Meningozele 


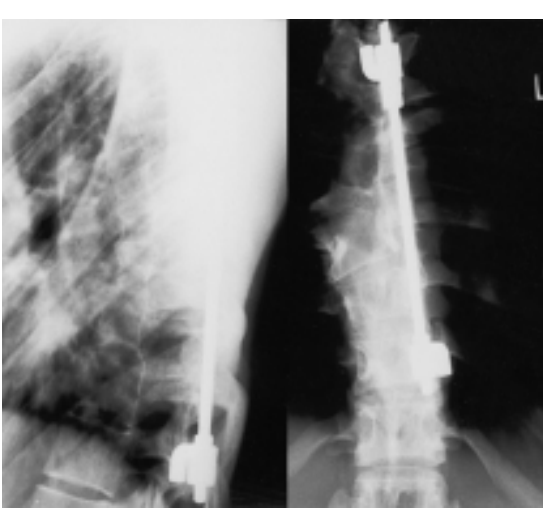

Abb. 1

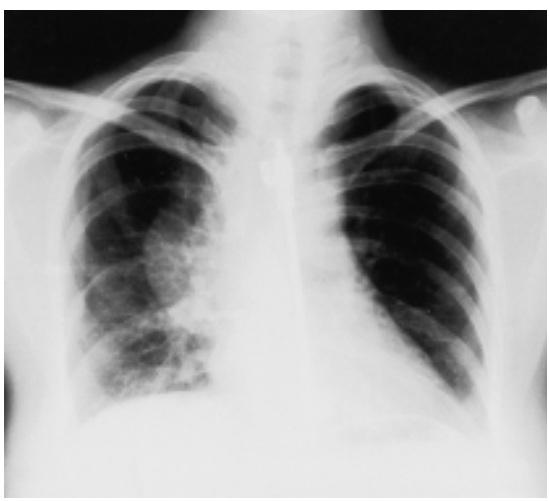

Abb. 2

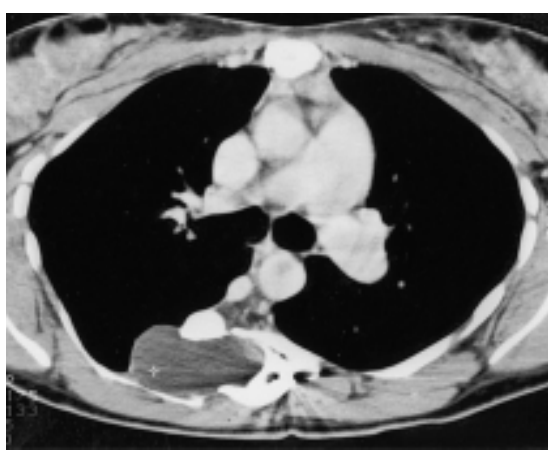

Abb. 3

rechts paravertebral ansammelte. Das anschließende Computertomogramm (Myelo-CT) zeigte einen ausgedehnten Defekt des Spinalkanals von BWK 5 bis BWK 8 rechtsseitig; es waren weder Foramina intervertebralia noch Querfortsätze abgrenzbar. Die Wirbelkörper waren erheblich abgeflacht, die Wirbelbögen verdickt und zur Gegenseite verschoben. Die Rippen 4 bis 9 rechts hatten keinen Kontakt mehr zu den Processi costotransversarii, sondern waren erheblich nach dorsal verlagert (Abb.4). Die coronaren Rekonstruktionen wiesen eine breite Verbindung zwischen Dural-

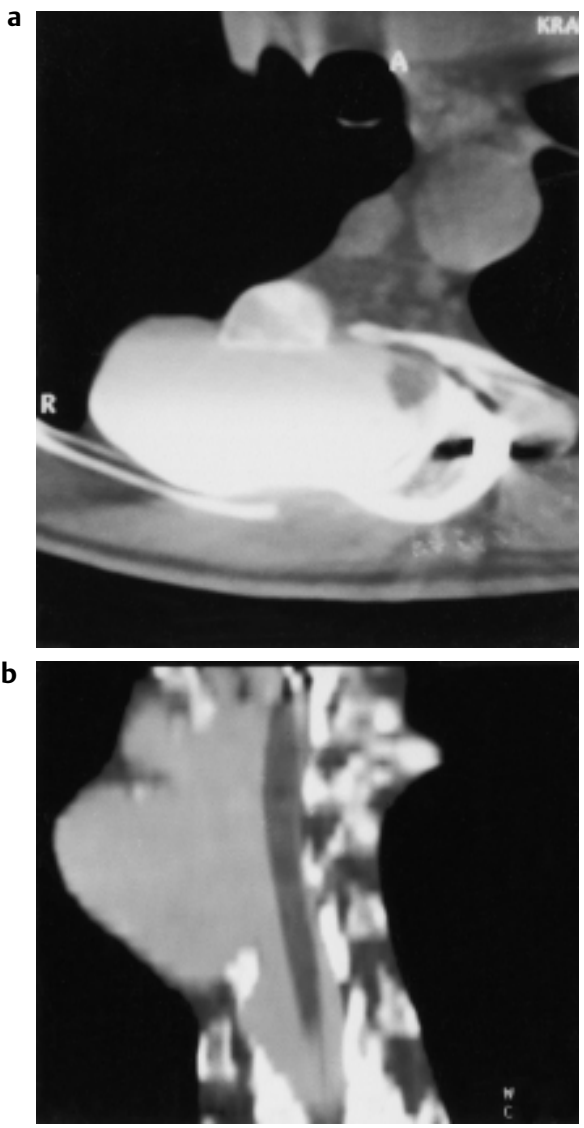

Abb. 4

sack und Meningozele nach, das Brustmark lag intraspinal und etwas zur Gegenseite verlagert. Es erfolgte die operative Abtragung der Zele mit Verschluss des Duralsackes. Postoperativ kam es zu einer langsamen Besserung der Schmerzsymptomatik.

\section{Diskussion}

Man unterscheidet zwei Formen der Neurofibromatose: Typ I (Morbus Recklinghausen) mit einem Defekt am langen Arm des Chromosom 17 und Typ II mit einem Defekt des Chromosom 22. Die Diagnose NF1 wird anhand von zwei oder mehr der folgenden Kriterien gestellt: Cafe au lait-Flecken, Neurofibrome oder plexiformes Neurofibrom, Lentigines der Axilla oder Leiste, Optikusgliom, Lisch-Knötchen der Iris, knöcherne Dysplasien, Verwandte ersten Grades mit NF 1. Kriterien der selteneren Neurofibromatose Typ II (NF 2) sind bilaterale Akustikusneurinome oder unilaterales Akustikusneurinom mit weiteren Veränderungen, meist intrakraniellen Tumoren.
Thorakale Meningozelen sind in 85\% der Fälle mit einer NF1 vergesellschaftet. Seltener findet man sie bei Bindegewebserkrankungen wie Marfan- oder Danlos-Ehlers-Syndrom oder als eigenes Krankheitsbild. Spinale Meningozelen bei Neurofibromatose werden durch mesodermale Dysplasien erklärt und entsprechen Herniationen des Duralsackes durch das Foramen intervertebrale oder durch ventrolaterale Knochenlücken. Sie sind in der Regel thorakal, selten lumbal oder zervikal gelegen (Rainov NG et al., Neurosurg Rev 1995; 18: 127-134). Im Gegensatz dazu liegen andere dysraphische Meningozelen in der Mittellinie, meistens lumbal und sakral mit Ausdehnung nach dorsal, seltener ventral.

Die laterale Meningozele erstreckt sich nach extrapleural in das hintere Mediastinum und kann von einer kleinen bis zu einer sehr ausgedehnten paravertebralen Raumforderung führen. Im Falle einer Skoliose der BWS liegt sie nahe des oberen Krümmungsscheitels und weist auf die konvexe Seite hin, in der Regel nach rechts. Bilaterale oder multiple intrathorakale Meningozelen kommen nur selten vor. Das Rückenmark liegt meist außerhalb des Zelensackes, gelegentlich verlaufen Nervenwurzeln durch die Zele.

Thorakale Meningozelen sind in der Hälfte der Fälle asymptomatisch. Rückenschmerzen werden von einem Viertel der Patienten angegeben, selten werden Parästhesien oder Paresen beobachtet. Eine Entscheidung zur operativen Sanierung ist abhängig von den neurologischen Ausfällen, der subjektiven Beeinträchtigung der Patienten und vom Ausmaß der thorakalen Raumforderung.

Diagnostisches Verfahren der Wahl ist die MRT, die eine flüssigkeitsgefüllte Raumforderung paravertebral mit einer Verbindung zum Spinalkanal aufzeigt. Oft wird jedoch zuerst in Röntgen-Übersichtsaufnahmen eine mediastinale Raumforderung festgestellt und die Abklärung erfolgt durch die CT oder MRT (Gut E et al., Radiologe 1996; 36: 453 455). Im vorliegenden Fall musste ein Myelo-CT durchgeführt werden, um eine ausreichende Darstellung der anatomischen Verhältnisse zu erreichen und die breite Kommunikation zwischen Duralsack und Zele aufzuzeigen. Dies ist ein erschwerender Faktor für die operative Versorgung im Gegensatz zu den Fällen, 
in denen nur ein Foramen intervertebrale erweitert ist (Zibis AH et al., Eur Radiology 2000; 10: 144-148).

Die Ätiologie von lateralen thorakalen Meningozelen wird unterschiedlich diskutiert. Nur wenig Beachtung finden Theorien, wonach es sich dabei um sekundär umgewandelte Neurofibrome oder um posttraumatische Veränderungen handelt. Eine operativ bedingte Verletzung der Meningen ist im vorliegenden Falle wegen der begleitenden Wirbelfehlbildungen und der breiten Kommunikation unwahrscheinlich. In der Literatur wird ursächlich eine primäre Dysplasie der Rückenmarkshäute diskutiert, die in Richtung der Nervenwurzeltaschen erweitert sind. Begünstigt durch den negativen intrathorakalen Druck und kontinuierliche Liquorpulsation kommt es zu einer Vergrößerung der Zele und zu sekundär zunehmenden knöchernen Defekten (Mai- uri F et al., Surg Neurol 1986; 26: 409 412). Diese Theorie wird durch Beobachtungen unterstützt, wo ein Vergleich mit früheren Röntgenaufnahmen das Auftreten oder die Vergrößerung von lateralen Meningozelen aufgezeigt hatte. Die häufige Assoziation mit einer Skoliose und knöchernen Anomalien weist jedoch auf eine gleichzeitige oder primäre Dysplasie der Wirbel hin (Maldonaldo RG et al., Zentralbl Neurochir 1992; 53: 11 14). Die Ausbildung einer Meningozele und ihre Größenzunahme kann auch hierbei durch sekundäre Faktoren erklärt werden. Im vorgestellten Fall sprechen der ausgedehnte Knochendefekt und die spätere Entwicklung einer Meningozele für eine primär mesodermale Fehlanlage der Wirbelsäule mit sekundärer Zelenbildung.

U. Dietrich, Bielefeld; O. Schneider, Bielefeld; I. Wanke, Essen 